\title{
A Study of Changing Trends in Potential Life-Partner Selection
}

Dr. Amena Zehra Ali ${ }^{1}$, Dr. Anjum Ara Jehangir ${ }^{2}$, Dr. Rakhshanda T. Hussain ${ }^{3}$, and Ms. Sheeza Rizwan ${ }^{4}$

\author{
${ }^{1}$ Associate Professor, Department of Psychology, University of Karachi \\ 2 Adjunct Professor, Department of Psychology University of Karachi, Pakistan \\ ${ }^{3}$ Research Fellow Department of Psychology University of Karachi, Pakistan \\ ${ }^{4}$ Research Fellow, Department of Psychology, University of Karachi, Pakistan
}

\begin{abstract}
Marriage is an important institution that upholds society's values. Thus an important decision for any adult is to select appropriate life partner. The definition of appropriateness is largely influenced by cultural norms and values. This research was conducted to study trends in potential mate preference characteristics of the Pakistani population. To attain the aforementioned objective, an archival research was conducted. Data was collected from Sunday matrimonial sections of two most widely read newspapers (Daily Jung and Daily Dawn). Entire data was drawn for 3 decades (i.e., 1980 - 2009). The requirements for to-be bride and to-be grooms were noted. Result showed that in both newspapers, Physical attractiveness in to-be brides was the top sought feature in three decades, whereas for to-be grooms, working or being educated were the most required traits. It shows that most of the requirements remained same, still few changes were noted within the three decades.
\end{abstract}

\section{Keywords}

mate selection, mate preference, archival, matrimonial, South Asia,

The sacred institution of marriage serves an important function of fulfilling a society's social and biological needs. The age old question of what qualities are considered appropriate in a potential mate is universal. The response to this question broadly differs from one society to another. Overall, the mate selection process from selection strategies to selection criteria is largely defined by cultural norms and values. An important research area in this regard is identification of the characteristics valued in a society while searching for potential mates. Haneef, Alvi and Ali (2014) replicated a Serbian study (Todosijević et al, 2003) with Pakistani university students and found out that attributes like faithfulness, neatness, physical attractiveness, beauty, talent for arts and courage were given importance as mate selection criteria. Another research with male and female undergraduates revealed the importance both placed on finding loving, caring and understanding spouses (Tariq, Hasan \& Ajmal, 2013).

Shoemake's (2007) preliminary review of three decade worth of research into human mate selection consistently revealed gender differences in preferences for mate characteristics (e.g., Buss, 1989; Buss \& Barnes, 1986; Cunningham, Barbee, \& Pike, 1990; Cunningham, 1986). Men tend to give more importance to physical attractiveness and youth (Fisman et al., 2006) while women value financial stability (Hitschet al., 2010) education, self-confidence, intelligence, dominance, and a higher social position than their own (Buunk et al., 2002). Men also tend to look for domestic and nurturing qualities (Howard et al. 1987). In one study by Botwin et al. (1997), preference for socially desirable personality traits was higher in women compared to men.

Other researchers have additionally examined changes in mate preferences over the years in the US culture. Hill and Chirstensen (1939) pioneered research in this area by a survey asking college students to rate 18 traits that were identified to be important in mate selection. Various replications of this study have been conducted to determine and explain any changes in the original findings with time (McGuinnis, R., 1959; Hudson and Henze, 1969; Hoyt and Hudson, 1981 and Buss and Barnes, 1986). A more recent quasi-replication in 2004 (O'Boyle \& Dawson, 2012) catered to certain methodological issues and came up with cotemporary counterparts of the original 18 trait list. Comparisons were made over the years with the original research and five subsequent replications (Buss et al., 2001), each from a decade leading up to 2004. Certain traits such as such as trustworthiness, good personality, attraction, and good health were still considered important 66 years later. However, intelligence and good looks were comparatively ranked higher by both males and females. The study further found that males rated traits like family orientation and cooking/housekeeping abilities lower than before while females gave less importance to finding a mate who is ambitious and on a similar education level as themselves. A research in India (Kamble et al., 2014) studied trends in mate preferences by comparing data gathered from an Indian sample in 1989 with data gathered from an Indian sample in 2009 consisting of both males and females. The importance of mutual attraction and love and sex differences in requirements signaling fertility and resources remained unchanged over time. Notable changes for both sexes included a higher preference for three qualities namely; creative and artistic, ambitious and industrious, and good cook and housekeeper.

Attempts have also been made to explore a set of universal dimensions underlying the extended lists of mate preference characteristics by factor analyzing the various ratings. Among the researches reviewed by Shackelford and colleagues (2005), both shorter and longer lists have been used for factor analyses. Simpson \& Gangestad (1992) identified Personal/Parenting Qualities and Attractiveness/Social Visibility after factor analyzing ratings of 15 characteristics. Previously Goodwin and Tang (1991) attempted the same and identified three dimensions: Kindness/Consideration, Extraversion, and Sensitivity. Factor analyses of ratings of over 75 attributes led Fletcher et al. (1999) to three dimensions namely Warmth-Trustworthiness, Vitality-Attractiveness, and Status- Resources. Shackelford et al. used an archival approach by utilizing data gathered from three dozen cultures (Buss, 1989) to identify four universal dimensions: Love vs. Status/ Resources; Dependable/Stable vs. Good Looks/Health; Education/Intelligence vs. Desire for Home/Children; and Sociability vs. Similar Religion. Consistent with previous literature sex differences were observed in three of the four 
dimensions with women valuing stability and education and men showing preference for good looks, health and nurturing characteristics.

The purpose of the present study is to identify the requirements people have in their minds when they are searching for the appropriate partner and whether this has changed over time. Particular to the Pakistani culture, various strategies are used in the process of searching for a partner. At times, a love marriage happens when the two partners have decided to marry after knowing each other for some time. Other times the family takes the responsibility of finding a suitable mate through word of mouth, referrals or professional marriage bureaus. This practice of arranged marriage is a common occurrence in South Asian cultures (Ibrahim, Ohnishi, and Sandu, 1997). A fairly common practice is to use electronic or print media. Newspaper matrimonial is one of the sources utilized for this purpose. This research will be using the content of these newspaper ads to identify preference characteristics in the Pakistani culture over the past 3 decades (1980 - 2009)

\section{Methodology}

\section{Sample}

Data collected from Sunday matrimonial sections of two popular newspapers: Daily Dawn (English) and Daily Jung (Urdu) served as the sampling frame for this study. The advertisements span over three decades i.e. 1980 to 2009 . The Jung of 1980 \&1982 and the Dawn of 1982 were unavailable.

\section{Material}

Material for the study included weekly matrimonial advertisements from two popular newspapers: Daily Dawn (English) and Daily Jung (Urdu). Newspapers were taken from Periodical section of Dr. Mehmood Hussain Library of University of Karachi. Online sources were utilized where possible. Advertisements specifying requirement criteria were selected.

\section{Procedure}

An initial survey was conducted to determine which newspapers are popularly approached for matrimonial ads after which Daily Dawn and Daily Jung were selected. The relevant sections of both the newspapers were collected and reviewed. The ones containing the requirements for brides to-be and grooms to-be were selected. From each advertisement, the requirement criteria were picked and frequency and percentages tables were developed from it. The initial review resulted in collection of hundreds of different characteristics that were required by people. In order to make the results manageable, similar characteristics were clustered together to form some major categories. Top three requirements were identified and compared for each newspaper across three decades (i.e. 1980-1989, 1990-1999 and 2000-2009).

\section{Results}

The list of requirement criteria gathered from the advertisements was clustered together under the following major categories: Physical appearance, Personality attributes, Education, Financial status, Green card/Foreign, Professional/Working, Ethnic background, Family background, Marital status, Religious sect and Miscellaneous. Further data analyses made use of percentages. Top three requirements were identified and compared for each newspaper across three decades (i.e. 1980-1989, 1990-1999 and 2000-2009).

Physical appearance remained the first ranked requirement for brides to-be across three decades in both newspapers. Requirements for Education and Personality attributes, which were ranked second and third respectively, were also seen to be consistent across both newspapers in the 2000's. The previous two decades showed some differences. In the 80's, education ranked second in the English newspaper and third in the Urdu newspaper. In the 90's, Education was completely replaced with Profession in second place and Marital status in third place. Personality attributes remained 2 nd most important in the Urdu newspaper for the first two decades

\section{Table 1: Top three ranks of the requirements in the three decades for brides to-be}

\begin{tabular}{lllllll}
\hline Rank & \multicolumn{2}{c}{ 1980-89 } & \multicolumn{2}{c}{ 1990-99 } & \multicolumn{2}{c}{ 2000-09 } \\
\cline { 2 - 6 } & \multicolumn{1}{c}{ Dawn } & Jung & Dawn & Jung & Dawn & Jung \\
\cline { 2 - 6 } $\mathbf{1}^{\text {st }}$ & Physical & Physical & Physical & Physical & Physical & Physical \\
& Appearance & Appearance & Appearance & Appearance & Appearance & Appearance \\
\multirow{2}{*}{$\mathbf{2}^{\text {nd }}$} & Education & Personality & Profession & Personality & Education & Education \\
& & Attributes & & Attributes & & \\
$3^{*}$ & \multirow{2}{*}{ Miscellaneous } & Education & Miscellaneous & Marital & Personality & Personality \\
& & & & Status & Attributes & Attributes \\
\hline
\end{tabular}

Common physical appearance requirements included fair, slim, tall, sharp features etc. Common personality attributes included well-mannered, domesticated, obedient, respectful, moralistic, social, mature, religious, understanding, 
compromising etc. Demands for education included the terms 'Educated' and 'Highly educated'. Specific to 90's, demands were made for a bride to-be with doctor as a profession.

Table 2: Percentages of requirement for brides to-be in the years 1980-89

\begin{tabular}{|c|c|c|c|c|c|c|c|c|c|c|c|}
\hline & & 1980 & 1981 & 1982 & 1983 & 1984 & 1985 & 1986 & 1987 & 1988 & 1989 \\
\hline \multirow{2}{*}{$\begin{array}{c}\text { Physical } \\
\text { Appearance }\end{array}$} & Dawn & 48.33 & 51.28 & ------ & 28.51 & 40.19 & 39.26 & 29.64 & 35.25 & 34.17 & 32.43 \\
\hline & Jung & ------ & 42.25 & 34.01 & 28.45 & 39.03 & 31.83 & 25.95 & 32.53 & 28.07 & 41.34 \\
\hline \multirow{2}{*}{$\begin{array}{l}\text { Personality } \\
\text { Attributes }\end{array}$} & Dawn & 4.91 & 5.31 & ------ & 11.13 & 9.14 & 10.16 & 19.76 & 9.13 & 23.57 & 9.04 \\
\hline & Jung & ------ & 12.38 & 16.03 & 30.52 & 15.34 & 22.32 & 27.51 & 10.58 & 24.98 & 18.45 \\
\hline \multirow[b]{2}{*}{ Education } & Dawn & 17.62 & 10.31 & ------ & 20.55 & 22.78 & 11.11 & 13.61 & 11.52 & 17.23 & 11.48 \\
\hline & Jung & ------ & 9.96 & 20.45 & 15.26 & 28.39 & 13.69 & 9.94 & 11.45 & 8.94 & 12.82 \\
\hline \multirow{2}{*}{$\begin{array}{c}\text { Financial } \\
\text { Status }\end{array}$} & Dawn & ------ & 10.88 & ------ & 4.57 & 0.24 & ------ & 2.96 & 7.3 & ------ & 0.56 \\
\hline & Jung & ------ & 14.11 & 9.09 & 3.22 & ------ & ------ & ------ & 10.77 & 4.13 & 6.87 \\
\hline \multirow{2}{*}{$\begin{array}{c}\text { Green } \\
\text { Card/Foreign }\end{array}$} & Dawn & 2.05 & 2.87 & ------ & 2.19 & 0.97 & 11.49 & 5.71 & 8.65 & ------ & 9.23 \\
\hline & Jung & ------ & ----- & ------ & 0.83 & 5.68 & 9.33 & 9.41 & 9.39 & 2.09 & ----- \\
\hline \multirow[b]{2}{*}{ Profession } & Dawn & 2.25 & ------ & ------ & ------ & 1.58 & 12.64 & 9.98 & 6.91 & ------ & 13.51 \\
\hline & Jung & ------ & 4.78 & 2.27 & ------ & ------ & 6.22 & ----- & 1.79 & 0.8 & ------ \\
\hline \multirow{2}{*}{$\begin{array}{c}\text { Ethnic } \\
\text { Background }\end{array}$} & Dawn & 8.81 & 13.18 & ------ & 6.35 & 18.51 & 6.23 & ------ & 2.88 & 6.26 & 8.56 \\
\hline & Jung & ------ & 3.81 & ------ & ------ & 5.17 & 4.66 & 9.19 & ------ & ------ & ------ \\
\hline \multirow{2}{*}{$\begin{array}{c}\text { Family } \\
\text { Background }\end{array}$} & Dawn & 7.73 & 6.16 & ------ & 8.64 & ------ & 9.09 & 13.23 & 10.37 & 18.76 & 10.81 \\
\hline & Jung & ------- & 12.68 & 19.13 & 10.69 & 11.15 & 7.39 & 9.35 & 10.26 & 11.84 & 10.15 \\
\hline \multirow{2}{*}{$\begin{array}{l}\text { Marital } \\
\text { Status }\end{array}$} & Dawn & ------ & ------ & ------ & 4.97 & ----- & ----- & ----- & 7.01 & ------ & 2.02 \\
\hline & Jung & ------ & ------ & ------ & 1.45 & ------ & ------ & ------ & 8.93 & 1.48 & 10.35 \\
\hline \multirow{2}{*}{$\begin{array}{c}\text { Religious } \\
\text { Sect }\end{array}$} & $\overline{\text { Dawn }}$ & ------ & ------ & ------ & ------ & ----- & ------ & ------ & ------ & ------ & ------ \\
\hline & Jung & ----- & ------ & ------ & 5.61 & ------ & ----- & & 2.29 & 12.95 & ------ \\
\hline \multirow[b]{2}{*}{ Misc. } & Dawn & ------ & ------ & ------ & ------ & ------ & ------ & ------ & 0.96 & ------ & 0.45 \\
\hline & Jung & ----- & ------ & ------ & 3.95 & 6.37 & ------ & ----- & 2.02 & 4.69 & ------ \\
\hline
\end{tabular}

As Table 2 shows overall the 80's showed a decline for the requirement criteria of physical appearance. Demands for personality attributes and green card holders showed an increase. Marital status and religious sect were given less importance over this decade showing an increase only in the last two years.

Table 3: Percentages of requirement for brides to-be in the years 1990-99

\begin{tabular}{|c|c|c|c|c|c|c|c|c|c|c|c|}
\hline & & 1990 & 1991 & 1992 & 1993 & 1994 & 1995 & 1996 & 1997 & 1998 & 1990 \\
\hline \multirow{2}{*}{$\begin{array}{c}\text { Physical } \\
\text { Appearance }\end{array}$} & Dawn & 34.14 & 42.53 & 33.07 & 21.85 & 12.56 & 28.83 & 39.81 & 42.38 & 51.99 & 32.76 \\
\hline & Jung & 37.49 & 39.62 & 29.67 & 20.27 & 14.26 & 34 & 35.71 & 27.11 & 33.73 & 35.86 \\
\hline \multirow{2}{*}{$\begin{array}{l}\text { Personality } \\
\text { Attributes }\end{array}$} & Dawn & 9.04 & 5.11 & 14.12 & 4.11 & 18.52 & 3.56 & 11.2 & 6.7 & 7.1 & 14.89 \\
\hline & Jung & 12.33 & 10.52 & 19.63 & 19.51 & 20 & 14.75 & 17.44 & 28.8 & 11.49 & 18.59 \\
\hline \multirow[b]{2}{*}{ Education } & Dawn & 11.48 & 11.73 & 12.64 & 6.35 & ------ & ------ & 10.19 & 10.23 & ------ & 6.91 \\
\hline & Jung & 25.5 & 17.99 & 18.6 & 8.99 & 5.42 & 6.04 & 7.37 & 13.01 & 9.89 & 11.52 \\
\hline
\end{tabular}




\begin{tabular}{|c|c|c|c|c|c|c|c|c|c|c|c|}
\hline \multirow{2}{*}{$\begin{array}{c}\text { Financial } \\
\text { Status }\end{array}$} & Dawn & 0.56 & 1.73 & 1.19 & 25.67 & 18.37 & ------ & ------ & ------ & 3.27 & 6.49 \\
\hline & Jung & 3.37 & ------ & 8.45 & 31.34 & 26.04 & ------ & 5.51 & ------ & 10.14 & 8.17 \\
\hline \multirow{2}{*}{$\begin{array}{c}\text { Green } \\
\text { Card/Foreign }\end{array}$} & Dawn & 9.23 & 10.98 & 6.12 & 1.77 & ------ & 5.68 & 8.43 & 10.34 & ------ & 11.38 \\
\hline & Jung & ------ & 6.78 & 2.63 & ------- & ------ & 7.96 & 3.33 & 5.95 & 5.56 & 3.16 \\
\hline \multirow[b]{2}{*}{ Profession } & Dawn & 13.51 & 11.73 & 11.94 & 22.59 & 25.57 & 24.45 & 5.55 & 7.95 & 18.47 & 15.64 \\
\hline & Jung & ------ & ------ & ------ & 10.42 & 17.05 & ------- & 5.71 & 3.13 & ------- & 7.62 \\
\hline \multirow{2}{*}{$\begin{array}{c}\text { Ethnic } \\
\text { Background }\end{array}$} & Dawn & 8.56 & 6.28 & 8.98 & 15.69 & 12.56 & 2.95 & ------ & ------ & ------ & 2.13 \\
\hline & Jung & ------ & 6.88 & 8.44 & ------ & ------- & ------ & ------ & 7.68 & 5.56 & ------ \\
\hline \multirow{2}{*}{$\begin{array}{c}\text { Family } \\
\text { Background }\end{array}$} & Dawn & 10.81 & 7.59 & 8.68 & ------ & ------- & 9.11 & ------ & ------ & 19.17 & ------ \\
\hline & Jung & 13.51 & 4.81 & 5.25 & ------ & ------ & 7.96 & 4.29 & 3.61 & 6.55 & 6.13 \\
\hline \multirow{2}{*}{$\begin{array}{l}\text { Marital } \\
\text { Status }\end{array}$} & Dawn & 2.02 & 0.99 & ------ & ------ & ------ & 18.36 & 20.8 & 15.33 & ----- & 0.96 \\
\hline & Jung & 4.05 & 1.57 & ------ & ------ & ------ & 26.32 & 15.57 & 5.17 & 17.06 & 1.12 \\
\hline \multirow{2}{*}{$\begin{array}{c}\text { Religious } \\
\text { Sect }\end{array}$} & Dawn & ------ & ------ & ------ & ------ & ------ & ------ & 3.98 & 7.16 & ------ & 5.31 \\
\hline & Jung & 3.71 & 7.76 & 7.31 & 9.47 & 10.85 & 4.57 & 3.49 & 4.55 & ------ & 7.11 \\
\hline \multirow[b]{2}{*}{ Misc. } & Dawn & 0.45 & 1.32 & 3.26 & 1.96 & 12.4 & ------ & ------ & ------ & ------ & ------ \\
\hline & Jung & ------ & 3.83 & ------ & ------- & 6.36 & ------ & 1.47 & 0.49 & ------- & ------- \\
\hline
\end{tabular}

Compared to the 80's, this decade (Table 3) showed an increase in importance of marital status and religious sect Specifically the mid 90's showed a marked increase in demands for financial status and ethnic background.

Table 4: Percentages of requirement for brides to-be in the years 2000-09

\begin{tabular}{|c|c|c|c|c|c|c|c|c|c|c|c|}
\hline & & 2000 & 2001 & 2002 & 2003 & 2004 & 2005 & 2006 & 2007 & 2008 & 2009 \\
\hline \multirow{2}{*}{$\begin{array}{c}\text { Physical } \\
\text { Appearance }\end{array}$} & Dawn & 22.96 & 22.41 & 24.84 & 22.4 & 25.91 & 30.23 & 29.67 & 29.3 & 24.03 & 17.4 \\
\hline & Jung & 33.14 & 10.59 & 17.95 & 20.78 & 23.05 & 23.6 & 19.21 & 32.91 & 18.72 & 23.21 \\
\hline \multirow{2}{*}{$\begin{array}{l}\text { Personality } \\
\text { Attributes }\end{array}$} & Dawn & 3.36 & 11.49 & 12.82 & 13.65 & 18.31 & 6.06 & 5.83 & 6.6 & 10.24 & 5.56 \\
\hline & Jung & 12.39 & 23.09 & 18.76 & 15.22 & 12.79 & 17.92 & 13.56 & 10.66 & 9.72 & 14.29 \\
\hline \multirow[b]{2}{*}{ Education } & Dawn & 24.97 & 11.06 & 19.69 & 17.6 & 16.62 & 16.03 & 14.42 & 20.62 & 18.45 & 26.11 \\
\hline & Jung & 5.8 & 15.06 & 11.43 & 12.68 & 8.4 & 14.87 & 13.16 & 6.85 & 13.24 & 11.06 \\
\hline \multirow{2}{*}{$\begin{array}{c}\text { Financial } \\
\text { Status }\end{array}$} & Dawn & 2.94 & 3.44 & 4.79 & 6.1 & 3.66 & 2.75 & 2.6 & 2 & 3 & 1.22 \\
\hline & Jung & 4.1 & 5.04 & 7.69 & 6.8 & 8.84 & 3.74 & 6.09 & 6.46 & 5.66 & 5.77 \\
\hline \multirow{2}{*}{$\begin{array}{c}\text { Green } \\
\text { Card/Foreign }\end{array}$} & Dawn & 5.46 & 5.46 & 8.1 & 7.56 & 7.42 & 6.27 & 7.01 & 6.3 & 5.93 & ------ \\
\hline & Jung & 1.34 & 0.33 & 4.54 & 5.35 & 0.77 & 0.82 & 2.89 & 4.05 & 3.06 & 2.61 \\
\hline \multirow[b]{2}{*}{ Profession } & Dawn & 9.84 & 11.21 & 10.97 & 11.3 & 9.4 & 13.11 & 8.2 & 4 & 8.15 & 6.31 \\
\hline & Jung & 7.43 & 9.51 & 5.97 & 7.2 & 5.87 & 6.09 & 6.48 & 4.24 & 10.41 & 6.62 \\
\hline \multirow{2}{*}{$\begin{array}{c}\text { Ethnic } \\
\text { Background }\end{array}$} & Dawn & 10.17 & 11.24 & 6.58 & 5.72 & 4.79 & 6.32 & 7.8 & 12.99 & 11.7 & 5.56 \\
\hline & Jung & 7.29 & 7.78 & 7.98 & 9.66 & 7.16 & 6.67 & 9.58 & 0.48 & 10.5 & 4.98 \\
\hline \multirow{2}{*}{$\begin{array}{c}\text { Family } \\
\text { Background }\end{array}$} & Dawn & ------ & ------ & ------ & ------ & ------ & ------ & ------ & ------ & ------ & ------ \\
\hline & Jung & ------ & ------ & ------ & ------ & ------ & ------ & ------ & ------ & ------ & ------ \\
\hline Marital & Dawn & 8.83 & 10.48 & 4.24 & 7.85 & 9.11 & 7.17 & 10.6 & 6.4 & 6.29 & 3.68 \\
\hline
\end{tabular}




\begin{tabular}{|c|l|r|r|r|r|r|r|r|r|r|r|}
\hline Status & Jung & 23.93 & 8.77 & 16.7 & 14.21 & 18.35 & 14 & 18.82 & 14.2 & 18.72 & 18.96 \\
\hline \multirow{2}{*}{$\begin{array}{c}\text { Religious } \\
\text { Sect }\end{array}$} & Dawn & 2.9 & 8.33 & 3.78 & 2.57 & 3.84 & 4.37 & 6.22 & 7.2 & 4.8 & 2.84 \\
\cline { 2 - 11 } & Jung & 1.06 & 5.29 & 3.29 & 3.18 & 4.82 & 5.97 & 4.32 & 4.58 & 5.07 & 4.31 \\
\hline \multirow{3}{*}{ Misc. } & Dawn & 7.8 & 3.01 & 5.19 & 4.18 & 8.6 & 6.61 & 6.3 & 3.6 & 6.7 & 4.5 \\
\cline { 2 - 11 } & Jung & 1.76 & 8.77 & 3.65 & 3.09 & 3.2 & 4.62 & 3.58 & 3.99 & 2.6 & 4.98 \\
\hline
\end{tabular}

Compared to the last two decades, the 2000's (Table 4) showed a slight decrease in the demands for physical appearance as demands for other requirements such as marital status, religious sect and ethnic background increased. Family background was given no importance at all.

Requirement for a job and financial status remained the first ranked for grooms to-be across three decades regardless of the newspaper. Education moved a rank upwards from the 80 's to being ranked second in the last two decades. Physical appearance requirements ranked third in the 80's and 90's before being replaced with personality attributes and ethnic background in the last decade.

\section{Table 5: Top three ranks of the requirements in the three decades for grooms to-be}

\begin{tabular}{cllcccc}
\hline \multirow{2}{*}{ Rank } & \multicolumn{2}{c}{ 1980-89 } & \multicolumn{2}{c}{ 1990-99 } & \multicolumn{2}{c}{ 2000-09 } \\
\cline { 2 - 6 } & \multicolumn{1}{c}{ Dawn } & \multicolumn{1}{c}{ Jung } & Dawn & Jung & Dawn & Jung \\
\cline { 2 - 6 } $\mathbf{1}^{\text {st }}$ & Finances/Working & Working & Working & Working & Working & Working \\
$2^{\text {nd }}$ & Miscellaneous & Miscellaneous & Education & Education & Education & Education \\
$\mathbf{3}^{\text {rd }}$ & Education & Physical & Physical & Physical & Personality & Ethnic \\
& & Appearance & Appearance & Appearance & Attributes & Background \\
& & & &
\end{tabular}

Common working requirements included running a business or having a profession as a doctor, engineer, government servant and armed forces. Medical, engineering and MBA degrees were required in education. Physical appearance requirements included handsome, smart, fair, healthy, tall etc. Common personality attributes included decent, respectful, broad-minded, good natured, moralistic, religious, compromising, mature, social etc.

Table 6: Percentages of requirement for grooms to-be in the years 1980-89

\begin{tabular}{|c|c|c|c|c|c|c|c|c|c|c|c|}
\hline & & 1980 & 1981 & 1982 & 1983 & 1984 & 1985 & 1986 & 1987 & 1988 & 1989 \\
\hline \multirow{2}{*}{$\begin{array}{c}\text { Physical } \\
\text { Appearance }\end{array}$} & Dawn & 15.85 & 24.97 & ------ & 23.29 & 6.21 & 16.19 & 10.09 & 15.89 & 7.44 & 3.1 \\
\hline & Jung & ----- & 19.94 & ------ & 15.8 & 6.26 & 18.18 & 13.46 & 12.92 & 12.47 & 8.21 \\
\hline \multirow{2}{*}{$\begin{array}{l}\text { Personality } \\
\text { Attributes }\end{array}$} & Dawn & 3.39 & 25.1 & ------ & 12.63 & 3.62 & 12.61 & 6.2 & 10.8 & 18.6 & 7.6 \\
\hline & Jung & ----- & 30.14 & ------ & 17.5 & 2.47 & 4.62 & ------ & 10.6 & 23.45 & 7.12 \\
\hline \multirow[b]{2}{*}{ Education } & Dawn & 15.47 & 24.22 & ------ & 18.96 & 27.46 & 10.49 & 11.54 & 13.68 & 9.57 & 9.88 \\
\hline & Jung & ------ & 7.37 & ------ & 22.39 & 27.09 & 16.94 & 27.89 & 12.62 & 2.67 & 21.89 \\
\hline \multirow{2}{*}{$\begin{array}{c}\text { Financial } \\
\text { Status }\end{array}$} & Dawn & 25.28 & ------ & ------ & 4.31 & 2.57 & ------ & 7.93 & 17.65 & 8.51 & 15.52 \\
\hline & Jung & ------ & ------ & ------ & 13.98 & 1.65 & 1.69 & ------ & 10.39 & 11.37 & ------ \\
\hline \multirow{2}{*}{$\begin{array}{c}\text { Green } \\
\text { Card/Foreign }\end{array}$} & Dawn & 4.15 & ------- & ------ & 3.44 & ------ & 10.62 & 11.39 & 2.86 & 1.95 & 4.22 \\
\hline & Jung & ------ & ------ & ------ & 1.6 & 0.24 & 5.13 & 9.1 & 2.52 & 0.71 & 2.74 \\
\hline \multirow[b]{2}{*}{ Profession } & Dawn & ------ & 15.96 & ------ & 6.6 & 16.72 & 21.63 & 43.41 & 11.03 & 4.07 & ------ \\
\hline & Jung & ------ & 17.51 & ------ & 2.61 & 29.21 & 36.22 & 34.03 & 20.05 & 2.66 & 23.72 \\
\hline \multirow{2}{*}{$\begin{array}{c}\text { Ethnic } \\
\text { Background }\end{array}$} & Dawn & 19.62 & 8.48 & ------ & 8.03 & 12.66 & 7.38 & ------ & 12.14 & 10.81 & 18.07 \\
\hline & Jung & ------ & 12.52 & ------ & 2.5 & 11.54 & ------ & 5.74 & ------ & ------ & ------ \\
\hline
\end{tabular}




\begin{tabular}{|c|l|l|r|r|r|r|r|r|r|r|r|}
\hline \multirow{2}{*}{$\begin{array}{c}\text { Family } \\
\text { Background }\end{array}$} & Dawn & 15.85 & 0.88 & ------ & 19.24 & 10.3 & 14.33 & 9.37 & 15.89 & 26.04 & 21.74 \\
\cline { 2 - 11 } & Jung & ------ & 5.1 & ------ & 9.55 & 10.25 & 7.18 & 5.62 & 16.75 & 13.88 & 5.47 \\
\hline \multirow{2}{*}{$\begin{array}{c}\text { Marital } \\
\text { Status }\end{array}$} & Dawn & ------ & ------ & ------ & ----- & 8.79 & ----- & ------ & ------ & 6.38 & ------ \\
\cline { 2 - 11 } & Jung & ------ & ------ & ------ & 1.25 & 5.41 & ------ & ------ & 10.99 & 1.09 & ------ \\
\hline $\begin{array}{c}\text { Religious } \\
\text { Sect }\end{array}$ & Dawn & ------ & ------ & ------ & ----- & ----- & ----- & ----- & ----- & ----- & ----- \\
\cline { 2 - 11 } & Jung & ------ & 7.42 & ------ & 8.75 & 5.77 & 8.87 & 4.15 & 1.22 & 28.39 & 26.27 \\
\hline \multirow{3}{*}{\begin{tabular}{c} 
Misc. \\
\cline { 2 - 11 }
\end{tabular}} & Dawn & ------ & ------ & ------ & 2.58 & 11.58 & 6.77 & ------ & ------ & 1.77 & 0.56 \\
\cline { 2 - 11 } & Jung & ------ & ------ & ------ & 4.09 & ----- & 1.17 & ------ & 1.93 & 9.49 & 4.38 \\
\hline
\end{tabular}

As shown in Table 6, requirements of particular religious sect showed a marked increase in the late 80's. The mid 80's showed an increase for demands of profession. Financial status showed a marked decrease till the mid 80's but increased later. Physical appearance requirements also decreased over the decade.

\section{Table 7: Percentages of requirement for grooms to-be in the years 1990-99}

\begin{tabular}{|c|c|c|c|c|c|c|c|c|c|c|c|}
\hline & & 1990 & 1991 & 1992 & 1993 & 1994 & 1995 & 1996 & 1997 & 1998 & 1990 \\
\hline \multirow{2}{*}{$\begin{array}{c}\text { Physical } \\
\text { Appearance }\end{array}$} & Dawn & 6.55 & 16.67 & 25.39 & 23.58 & 12.77 & 13.1 & 28.36 & 16.38 & 12.75 & 3.46 \\
\hline & Jung & 8.52 & 15.92 & 11.03 & 0.26 & 14.85 & 14.91 & 12.16 & 21.12 & 8.92 & 13.77 \\
\hline \multirow{2}{*}{$\begin{array}{l}\text { Personality } \\
\text { Attributes }\end{array}$} & Dawn & 6.98 & 0.43 & ------ & ------ & 31.77 & 14.16 & 12.17 & 11.34 & 10.35 & 13.47 \\
\hline & Jung & 12.61 & 7.59 & 3.17 & 4 & 9.55 & 14.03 & 9.94 & 13.63 & 22.37 & 11.23 \\
\hline \multirow[b]{2}{*}{ Education } & Dawn & 16.15 & 13.95 & 15.53 & ------ & 4.63 & 14.17 & 14.58 & 13.86 & 20.27 & 20.8 \\
\hline & Jung & 20.44 & 10.43 & 11.62 & ------ & ------ & 21.05 & 17.17 & 13.63 & 15.31 & 15.21 \\
\hline \multirow{2}{*}{$\begin{array}{c}\text { Financial } \\
\text { Status }\end{array}$} & Dawn & 10.48 & 11.79 & 11.81 & 13.53 & 20.12 & 0.78 & ------ & 10.08 & 12.16 & 14.05 \\
\hline & Jung & ------ & 13.43 & 16.08 & 15.33 & 20.16 & 7.02 & 2.14 & ------ & 7.72 & 2.17 \\
\hline \multirow{2}{*}{$\begin{array}{c}\text { Green } \\
\text { Card/Foreign }\end{array}$} & Dawn & 4.36 & 16.97 & 5.17 & 6.76 & ------ & ------ & 3.04 & ------ & ------ & 4.77 \\
\hline & Jung & 2.04 & 3.7 & 1.76 & 5.47 & 3.18 & ------ & ------ & ------ & ------ & ------ \\
\hline \multirow[b]{2}{*}{ Profession } & Dawn & 21.83 & 28.9 & 27.18 & 44.47 & 22.67 & 51.96 & 31.39 & 29.96 & 33.02 & 22.57 \\
\hline & Jung & 21.12 & 17.22 & 15.14 & 60.53 & 27.59 & 40.53 & 47.91 & 33.39 & 28.76 & 22.83 \\
\hline \multirow{2}{*}{$\begin{array}{c}\text { Ethnic } \\
\text { Background }\end{array}$} & Dawn & 17.9 & 2.87 & 6.14 & 11.6 & 7.98 & ------ & 6.4 & 14.84 & 7.65 & ------ \\
\hline & Jung & ------ & 2.96 & 7.28 & ------ & ------ & 0.7 & ------ & 5.45 & 3.33 & 6.88 \\
\hline \multirow{2}{*}{$\begin{array}{c}\text { Family } \\
\text { Background }\end{array}$} & Dawn & 15.28 & 5.75 & 8.09 & ------ & ------ & ------ & ------ & ------ & 3.75 & 8.43 \\
\hline & Jung & 8.86 & 8.79 & 9.04 & ------ & ------ & ------ & ------ & ------ & ------ & 7.07 \\
\hline \multirow{2}{*}{$\begin{array}{l}\text { Marital } \\
\text { Status }\end{array}$} & Dawn & ------ & ------ & 0.64 & ------ & ------ & 3.8 & 4.01 & 3.5 & ------ & 0.84 \\
\hline & Jung & ------ & ------ & ------ & 2.93 & 12.73 & 1.75 & 5.19 & 3.92 & 0.13 & 0.54 \\
\hline \multirow{2}{*}{$\begin{array}{c}\text { Religious } \\
\text { Sect }\end{array}$} & Dawn & ------ & ------ & ------ & ------ & ------ & 1.96 & ------ & ------ & ------ & 9.55 \\
\hline & Jung & 25.21 & 16.67 & 17.72 & 6.67 & ------ & ------ & 3.06 & 8.86 & 4.79 & 9.96 \\
\hline \multirow[b]{2}{*}{ Misc. } & Dawn & ------ & ------ & ------ & ------ & ------ & ------ & ------ & ------ & ------ & 1.96 \\
\hline & Jung & 1.19 & 1.85 & 5.99 & 2.13 & 10.61 & ------ & 2.41 & ------ & 8.66 & 0.18 \\
\hline
\end{tabular}

The 90's showed a marked decrease for religious sect requirements before increasing slightly at the end. The groom's tobe profession gained much importance in 1993 and 1995 before declining. 
Table 8: Percentages of requirement for grooms to-be in the years 2000-09

\begin{tabular}{|c|c|c|c|c|c|c|c|c|c|c|c|}
\hline & & 2000 & 2001 & 2002 & 2003 & 2004 & 2005 & 2006 & 2007 & 2008 & 2009 \\
\hline \multirow{2}{*}{$\begin{array}{c}\text { Physical } \\
\text { Appearance }\end{array}$} & Dawn & 11.37 & 8.73 & 2.35 & 3.42 & 5.26 & 2.9 & 3.5 & 4 & 4.04 & 6.34 \\
\hline & Jung & 5.77 & 1.1 & 5.72 & 2.79 & 2.11 & 2.08 & 1.88 & 2.61 & 0.4 & 1.21 \\
\hline \multirow{2}{*}{$\begin{array}{l}\text { Personality } \\
\text { Attributes }\end{array}$} & Dawn & 13.01 & 9.51 & 9.88 & 15.08 & 3.11 & 4.97 & 10.9 & 6.37 & 7.73 & 7.28 \\
\hline & Jung & 10.34 & 11.42 & 13.24 & 14.13 & 7.93 & 7.13 & 9,49 & 5.64 & 14.84 & 8.48 \\
\hline \multirow[b]{2}{*}{ Education } & Dawn & 20.9 & 18.44 & 17.4 & 19.51 & 14.82 & 15.88 & 18 & 16.8 & 22.71 & 19.96 \\
\hline & Jung & 19.23 & 21.01 & 15.75 & 14.6 & 14.02 & 17.1 & 18.42 & 20.57 & 14.81 & 25.02 \\
\hline \multirow{2}{*}{$\begin{array}{c}\text { Financial } \\
\text { Status }\end{array}$} & Dawn & 3.49 & 5.24 & 7.55 & 10.13 & 6.12 & 1.79 & 5.9 & 3.55 & 7.85 & 3.17 \\
\hline & Jung & 0.91 & 0.92 & 4.52 & 0.64 & 4.03 & 4.68 & 1.5 & 7.69 & 4.62 & 2.59 \\
\hline \multirow{2}{*}{$\begin{array}{c}\text { Green } \\
\text { Card/Foreign }\end{array}$} & Dawn & 7.88 & 6.79 & 15.48 & 5.44 & 2.25 & 3.31 & 6.9 & 3.7 & 5.83 & 4.48 \\
\hline & Jung & 3.19 & 1.38 & 2.45 & 2.35 & 6.78 & 2.67 & 1.44 & 1.74 & 5.46 & 2.1 \\
\hline \multirow[b]{2}{*}{ Profession } & Dawn & 21.23 & 15.72 & 22.86 & 23.95 & 26.31 & 23.89 & 20.5 & 25.03 & 21.05 & 28.54 \\
\hline & Jung & 24.71 & 6.63 & 23.6 & 27.2 & 18.56 & 25.42 & 18.93 & 22.83 & 19.77 & 27.27 \\
\hline \multirow{2}{*}{$\begin{array}{c}\text { Ethnic } \\
\text { Background }\end{array}$} & Dawn & 3.41 & 10.07 & 4.27 & 3.92 & 7.94 & 20.85 & 8.7 & 9.48 & 10.34 & 6.16 \\
\hline & Jung & 16.27 & 5.34 & 11.88 & 12.54 & 19.97 & 7.95 & 20.56 & 18.36 & 11.48 & 11.69 \\
\hline \multirow{2}{*}{$\begin{array}{c}\text { Family } \\
\text { Background }\end{array}$} & Dawn & ----- & ------ & ------ & ------ & ------ & ------ & ------ & ------ & ------ & ------ \\
\hline & Jung & ------ & ------ & ------ & ------ & ------ & ------ & ------ & ------ & ------ & ------ \\
\hline \multirow{2}{*}{$\begin{array}{l}\text { Marital } \\
\text { Status }\end{array}$} & Dawn & 3.74 & 11.65 & 3.68 & 7.85 & 7.19 & 2.62 & 4.5 & 4.74 & 4.39 & 7.84 \\
\hline & Jung & 3.19 & 4.42 & 10.85 & 9.95 & 10.62 & 10.78 & 11.25 & 7.02 & 12.23 & 6.23 \\
\hline \multirow{2}{*}{$\begin{array}{c}\text { Religious } \\
\text { Sect }\end{array}$} & Dawn & 2.36 & 8.15 & 4.71 & 3.92 & 6.55 & 8.7 & 6.6 & 9.18 & 4.88 & 5.59 \\
\hline & Jung & 3.42 & 5.34 & 5.28 & 6.06 & 5.56 & 7.95 & 3.96 & 2.05 & 5.89 & 7.62 \\
\hline \multirow[b]{2}{*}{ Misc. } & Dawn & 2.69 & 1.55 & 10.47 & 13.94 & 6.22 & 14.22 & 12.9 & 12.88 & 9.16 & 8.77 \\
\hline & Jung & 6.38 & 4.52 & 3.16 & 4.6 & 7 & 6.15 & 7.79 & 6.25 & 4.5 & 6.49 \\
\hline
\end{tabular}

Compared to the first two decades, demands of physical appearance for grooms to-be have shown a marked decrease. Requirements for family background decreased to none.

\section{Discussion}

The results of the study indicate a consistent pattern of mate selection requirement over three decades, for both males and females. Regardless of the newspaper, attractive physical features remained one of the top most requirements for brides to-be. The same was true for the requirement of holding a job or business in grooms to-be. The finding is consistent with previous literature (Buss et al., 2001) which shows that men tend to attach greater importance to physical attractiveness of women, whereas women give more importance to good financial prospects. Much similar to our culture, a research in India also revealed a preference for beautiful brides and financially stable grooms (Shipman, 2010). Preference for financially stable men who can provide the necessary resources seems to be a universal requirement for women, with its strength varying depending on the culture. It is strongest in cultures where women have least opportunity to get an education, hold a job, or accumulate property of their own (Kasser \& Sharma, 1999).

On the other hand, Buss \& Schmitt (1993) reported that men, no matter how they look, always prefer a physically attractive partner. In the Pakistani culture, mothers play a significant role in inculcating this mindset in their sons, since the very beginning. They seek physical attractiveness in their future daughter-in-laws for the sake of ensuring a beautiful future generation. Additionally, physically attractive people benefit from a phenomenon referred to as the 'halo effect' (Dion, Berscheid, \& Walster, 1972). They are assumed to possess other desirable social characteristics such as warmth, kindness, poise, good character (Benokraitis, 2011), better physical and mental health (Langlois et al., 2000) and high moral values (Darby \& Jeffers, 1988). Research has also shown gender difference in the physical attractiveness stereotype with men basing more of their positive evaluations on a woman's attractiveness as compared to women's evaluation of attractive men (Berry \& Miller, 2001). This has created a social pressure on women as one global survey revealed about $59 \%$ of women 
strongly agree "physically attractive women are more valued by men" (Etcoff et al., 2004). Perhaps this strengthens the halo effect in the way that women possessing the ideal 'attractive' features grow up more confident as compared to those who are not. This confidence shows in their social dealings portraying a favorable image to others. There is evidence that physically attractive people are more likely to behave positively in social interactions (Langlois et al., 2000).

Overall the research literature has used two perspectives to explain these recurring gender differences. The evolutionary perspective with its roots in the basic principles of Darwin (1859, 1852, as cited in French et al., 2014) assumes that current mate choices are continually influenced by historically successful mate choices (Kenrick, 1994) that ensured the survival of our species. Based on this, the parental investment model (Trivers, 1972) further suggests that sexual differentiation in mate selection has occurred because men and women are required to invest different resources to rear their offspring. They tend to look for characteristics that indicate chances of successful child rearing. Hence, men prefer physical attractiveness and youth because it indicates a woman's reproductive capacity (Rhodes, 2006) while women prefer financial stability and status as a cue for a man's ability to obtain needed resources (Bryan et al., 2011).

On the other hand the social structural perspective proposes that these differences arise from the differing social roles that men and women occupy in a society (Eagly and Wood, 1999). These roles define their societal obligations and responsibilities. In a culture where a man is seen as bread winner and a woman as seen as a home maker, both would benefit by searching for characteristics that ensure these roles can be fulfilled. Shoemake (2007) uses the term 'marketable exchange' for this perspective. With respect to the Pakistani culture as in many others, men are assigned the typical social roles of power, dominance and financial independence. Women can access financial resources by seeking such characteristics in potential mates in exchange for their physical beauty and fertility. Men, on the other hand, market these very characteristics to seek out partners possessing characteristics that they desire.

Further data analysis sheds light on the nature of the Pakistani mindset regarding mate selection criteria. Over all there seems to be some similarity in the qualities that were asked for in the matrimonial ads. They just vary in proportion depending on the gender. Physical appearance features that were specified for women such as fair, slim, tall, sharp features, healthy and beautiful also appeared in the ads for men. Comparatively more emphasis was placed on height for men. Previous researchers have found taller men are considered more attractive as oppose to those with an average height (Hensley, 1994) perhaps because height is associated with dominance and power. In contrast, fairness and thinness was emphasized for women. The practice of equating light skin color with beauty is not new in the South-Asian culture. Its roots can be traced back to the British colonialism when the true disparity between the lighter skinned upper class and the darker skinned lower class was created. Since then the South-Asian culture has been under much influence of Western ideals. This is even reflected in typical fairness cream ads where a darker young woman faces rejection from potential mates before turning heads wherever she goes after obtaining a lighter skin color. The media industry plays a vital role in promoting and maintaining the physically attractive ideals for both men and women. Despite campaigns that are working to break these stereotypes, our results show that mindsets will require more time to change. Since newspaper advertisements maintain anonymity it is much easier to place certain demands that would not be spoken of out loud.

Similarly, personality attributes for both are not so different either. Both parties sought attributes like good nature, respectful, honest, mature, moralistic, compromising, understanding, social and religious. The exception for women included obedient and domesticated which again reflects a culture where a woman is required to prioritize her household over other things. Another interesting finding is that majority of the time the profession of a doctor was preferred in a bride-to-be. This contradicts the requirement for a domestic attribute and gives support for a popular notion that mothers want their daughter in-laws to be doctors by profession only so that they can bring a good name to their family. Similarly bringing in a physically attractive daughter-in-law into the family may reflect a desire to possess something beautiful just as one wants the best of materialistic possessions. According to Winegard and colleagues (2013) the tendency to flaunt attractive mates may serve to signal information regarding ones status and desirability, just as possessing and flaunting a luxury good does.

Religiosity was a fairly common requirement for both. This is perhaps because religiosity is assumed to be associated with good personal qualities and moral character. Research has previously shown that basic religious beliefs have a positive correlation with good characteristics that can ensure better adjustment and mental health (Khoynezhad et al., 2012). An increase in demands for specific ethnic and religious sects may reflect a growing disparity and intolerance within the culture.

\section{Conclusion}

The findings of the study have revealed how little has changed over three decades in the Pakistani population's mindset regarding mate selection requirements. Women are still required to possess physical attractiveness ideals and men are required to hold a position that ensures their financial stability. Other requirements showed fluctuations over time. Education and personality attributes gained more importance.

\section{References}

1. Benokraitis, N.V. (2011). Marriages \& Families: Changes, Choices, and Constraints. 7th edition. New Delhi: PHI Learning.

2. Berry, D., \& Miller, K. (2001). When boy meets girl: Attractiveness and the five-factor model in opposite-sex interactions. Journal of Research in Personality, 35, 62-77.

3. Botwin, M.D., Buss, D.M., \& Shackelford, T.K. (1997). Personality and Mate Preferences: Five Factors In Mate Selection and Marital Satisfaction. Journal of Personality, 65(1), 107-136.

4. Bryan, A.D., Webster, G.D., \& Mahaffey, A.L. (2011). The big, the rich, and the powerful: physical, financial, and social dimensions of dominance in mating and attraction. Personality and Social Psychology Bulletin, 37, 365-382. 
5. Buss , D.M. \& Schmitt, D.P. (1993). Sexual strategies theory: An evolutionary perspective on human mating. Psychological Review, 100, 204-232.

6. Buss, D. M. (1989). Sex differences in human mate preferences: Evolutionary hypotheses tested in 37 cultures. Behavioral and Brain Sciences, 12, 1-49

7. Buss, D. M., \& Barnes, M. (1986). Preferences in human mate selection. Journal of Personality and Social Psychology, 50, 559-570.

8. Buss, D., Shackelford, T., Kirkpatrick, L., and Larson, R. (2001). A Half- Century of Mate Preferences: the cultural evolution of values. Journal of Marriage and the Family, 63 (2), 491-504

9. Buunk, B. P., Dijkstra, P., Fetchenhauer, D. and Kenrick, D. T. (2002), Age and Gender Differences in Mate Selection Criteria for Various Involvement Levels. Personal Relationships, 9, 271-278. doi:10.1111/1475 6811.00018

10. Cunningham, M. R. (1986). Measuring the physical in physical attractiveness: Quasi- experiments on the sociobiology of female facial beauty. Journal of Personality and Social Psychology, 50, 925-935.

11. Cunningham, M. R., Barbee, A. P. \& Pike, C. L. (1990). What do women want? Facialmetric assessment of multiple motives in the perception of male facial physical attractiveness. Journal of Personality and Social Psychology, 59, $61-72$

12. Darby, B. W., \& Jeffers, D. (1988). The effects of defendant and juror attractiveness on simulated courtroom decisions. Social Behavior and Personality, 16, 39-50.

13. Dion, K., Berscheid, E., \& Walster, E. (1972). What is beautiful is good. Journal of Personality and Social Psychology, 24, 285-290

14. Eagly, A. H., \& Wood, W. (1999). The origins of sex differences: Evolution versus social structure. American Psychologist, 54, 408-423.

15. Etcoff, N., Orbach, S., Scott, J., \& D'Agostino, H. (2004). The Real Truth About Beauty: A Global Report.

16. Fisman, R., lyengar, S.S., Kamenica, E., Simonson, I., 2006. Gender differences in mate selection: evidence from a speed dating experiment. Quarterly Journal of Economics. 121, 673-697.

17. Fletcher, G. J. O., Simpson, J. A., Thomas, G., \& Giles, L. (1999). Ideals in romantic relationships. Journal of Personality and Social Psychology, 76, 72-89.

18. French, M.T., Popovici, I., Robins, P.K., \& Homer, J.F. (2014). Personality traits, cohabitation and marriage. Social Science Research, 45, 184-199. http://dx.doi.org/10.1016/j.ssresearch.2014.01.002

19. Goodwin, R., \& Tang, D. (1991). Preferences for friends and close relationship partners: A cross-cultural comparison. Journal of Social Psychology, 131, 579-581.

20. Haneef, M., Alvi, A.K., Ali, F. (2014). Spouse Selection Criteria : A Case Study Of University Students. Science International, 26(5), 2509-2513,

21. Hensley, W. E. (1994). Height as a basis for interpersonal attraction. Adolescence, 29, 469-474.

22. Hill, R. (1945). Campus Values in Mate Selection. Journal of Home Economics. Nov. pp. 554-558

23. Hitsch, G.J., Hortacsu, A., Ariely, D., 2010. What makes you click? - Mate preferences in online dating. Quantitative Marketing and Economics. 8, 393-427

24. Howard, J. A., Blumstein, P., \& Schwartz, P. (1987). Social or evolutionary theories: Some observations on preferences in human mate selection. Journal of Personality and Social Psychology, 53, 194-200.

25. Hoyt, Les and John Hudson (1981). Personal Characteristics Important in Mate Preference Among College Students. Social Behavior and Personality, 9 (1), 93-96.

26. Hudson, J.W. \& Henze, L.F. (1969) Campus value in mate selection: A replication. Journal of Marriage and the Family, 31 (Nov), 772-75

27. Ibrahim, F., Ohnishi, H., \& Sandhu, D. S. (1997). Asian American identity development: a culture specific model for South Asian Americans. Journal of Multicultural Counseling and Development, 25, 34-50

28. Kamble, S., Shackelford, T. K., Pham, M., \& Buss, D. M. (2014). Indian mate preferences: Continuity, sex differences, and cultural change across a quarter of a century. Personality and Individual Differences, 70, 150-155

29. Kasser, T. \& Sharma, Y.S. (1999). Reproductive freedom, educational quality, and females' preference for resource acquisition characteristics in mates. Psychological Science, 10, 374-377.

30. Kenrick, D. T. (1994). Evolutionary social psychology: From sexual selection to social cognition. In M. P. Zanna (Ed.), Advances in Experimental Social Psychology: Vol. 26 (pp. 75-121). San Diego: Academic Press

31. Khoynezhad, G., Rajaei, A. R., \& Sarvarazemy, A. (2012). Basic Religious Beliefs and Personality Traits. Iranian Journal of Psychiatry, 7(2), 82-86.

32. Langlois, J. H., Kalakanis, L., Rubenstein, A. J., Larson, A., Hallam, M., \& Smoot, M. (2000). Maxims or myths of beauty? A meta-analytic and theoretical review. Psychological Bulletin, 126, 390-423.

33. McGinnis, R. (1959). Campus Values in Mate Selection: a repeat study. Social Forces. May, pp. 368-373

34. O’Boyle, T., \& Dawson, J. (2012). College Campus Mate Selection: A Quasi-Replication. American International Journal of Social Science, 1(2), $1-11$.

35. Rhodes, G., (2006). The evolutionary psychology of facial beauty. Annual Review of Psychology, 57, 199-226

36. Shackelford, T.K., Schmitt, D.P., \& Buss, D.M. (2005). Universal dimensions of human mate preferences. Personality and Individual Differences, 39, 447-458.

37. Shipman, A. C., (2010). Mate Selection in Modern India. Doctoral Dissertations.

38. Shoemake, E.G. (2007). Human mate selection theory: An integrated evolutionary and social approach. Journal of Scientific Psychology, 35-41.

39. Simpson, J. A., \& Gangestad, S. W. (1992). Sociosexuality and romantic partner choice. Journal of Personality, $60,31-51$. 
40. Tariq, I. I., Hasan, S. S., \& Ajmal, M. A. (2013). Cognitive Schemas of an Ideal Spouse among Young Adults. Pakistan Journal of Social and Clinical Psychology, 11(2), 14-21.

41. Todosijević B., Ljubinković S., Arančić A., (2003). Mate selection criteria: A trait desirability assessment study of sex differences in Serbia. Evolutionary Psychology, 1, 116-126.

42. Trivers, R., (1972). Parental investment and sexual selection. In: Campbell, B. (Ed.), Sexual Selection and the Descent of Man, 1871-1971. Aldine, Chicago, pp.136-179

43. Winegard, B. M., Winegard, B., Geary, D, C. (2013). If You've Got It, Flaunt It: Humans Flaunt Attractive Partners to Enhance Their Status and Desirability. PloS ONE, 8. doi:10.1371/journal.pone.0072000

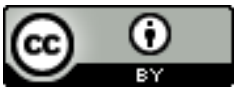

This work is licensed under a Creative Commons Attribution 4.0 International License.

DOI: $10.24297 /$ jssr.v11i2.6053 